\title{
The neurosensory musculocutaneous tensor fasciae latae flap: long term results
}

\author{
W Kuhn MD,${ }^{1} \mathrm{~N}$ J Lüscher MD,${ }^{2} \mathrm{R}$ de Roche MD,${ }^{2} \mathrm{~S}$ Krupp MD,${ }^{3} \mathrm{G}$ A Zäch ${ }^{4}$
}

${ }^{1}$ Praxis für Paraplegiologie, CH-6988 Ponte Tresa, Switzerland; ${ }^{2}$ Klinik für Plastische und Wiederherstellungschirurgie der Universität, Kantonsspital, CH-4031 Basel, Switzerland; ${ }^{3}$ Service de Chirurgie Plastique et Reconstructive CHUV, CH-1011 Lausanne, Switzerland; ${ }^{4}$ Schweizerisches Paraplegikerzentrum, CH-4055 Basel, Switzerland.

In 1971 we started covering pressure sores and unstable scars with transpositionrotation-muscle and musculocutaneous flaps. In 1980 we published the first results with 6 neurosensory musculocutaneous tensor fasciae latae flaps. ${ }^{1}$ Until April 1989, 31 tensor fasciae latae flaps (TFL) were used, and we review a consecutive series of 19 neurosensory TFL-flaps. Questions such as whether to delay the procedure; early and late complications; evolution of the sensation; and indications are outlined under the aspects of long term follow up studies. The conclusion is that if the neurological pattern permits a neurosensory flap, such flaps should be done because no local recurrence occurred. In extended neurosensory TFL-flaps sensation of the filling status of the rectum is improved, and sitting control and perception of the 'body scheme' are also improved.

Key words: ischial and trochanteric pressure sores; neurosensory musculocutaneous tensor fasciae latae flaps; spinal cord lesions.

\section{Introduction}

Soft tissue breakdown still often occurs in patients with spinal cord lesions, and remains the main cause for readmissions to a paraplegic centre. ${ }^{2}$ Risk factors such as reduced mobility (physical and psychological), loss or decrease in vasomotor control, alteration in anatomy and last but not least the loss of protective sensation are sometimes difficult to eliminate. ${ }^{1-5}$

Bringing protective sensation and adequate padding to the typical pressure sore sites around the pelvis therefore appears to be an important aim, not only for treatment but also for the prevention of pressure sores. ${ }^{1,6-11}$

After early experiences with neurosensory flaps such as the sensory intercostal flap used by Dibbel in 1974, ${ }^{12}$ Nahai and other authors $1,6,11.13$ reported the use of the neurosensory tensor fasciae latae musculocutaneous flap. This flap offers the possibil-

Correspondence: Swiss Paraplegic Centre Balgrist, CH-8008 Zurich, Switzerland. ity of bringing sensory skin from the lateral aspect of the thigh to the ischial and/or trochanteric region in patients with spinal cord lesions below L3. Up to now only small series with short or incomplete follow up studies have been published.

\section{Methods}

Anatomical and surgical considerations

The tensor fasciae latae flap is a very reliable transposition or island flap vascularised by the lateral circumflex femoral artery, which enters the muscle on its deep surface approximately $8 \mathrm{~cm}$ distal to the anterior superior iliac spine. ${ }^{13}$ The skin of the lateral thigh is vascularised by musculocutaneous, and distally by fasciocutaneous, perforating vessels from the fasciae latae. The distal third of an extended TFL-flap has a supplementary vascular supply from posterior perforators from the deep femoral artery, and anterior musculocutaneous perforators from the vastus lateralis muscle. ${ }^{14}$ Therefore this part of the flap is almost a random pattern flap. 
In patients with a spinal cord lesion below L3 the lateral femoral cutaneous nerve is intact, and the whole lateral aspect of the thigh down to the knee joint is covered with sensory skin that allows the transfer of the extended TFL-flap as a sensory unit.

Few cases are reported where the lateral femoral cutaneous nerve was neurotised by nerve grafting. ${ }^{4.8 .10 .15}$

The flap may be raised as a lateral or medial transposition flap or as a medial island flap. ${ }^{1.11}$ The advantage of the lateral transposition is the possibility of covering and protecting the trochanter with neurosensory musculocutaneous flaps.

Extended medial island flaps with a deepithelialised 'musculo-fascio-dermal' pedicle for coverage of the ischial tuberosity can be used as an additional possibility.

For a safe transfer, especially in obese patients or in patients with skeletal deformities, Krupp ${ }^{16}$ advocates delay of the distal part of the flap.

In doubtful cases, ie in patients with multiple scars in the lateral pelvic regions (such as spina bifida patients with hip correction operations), a preoperative angiogram can show a patent lateral circumflex femoral artery (Fig 1).

\section{Indication}

Generally speaking plastic surgery should only be considered in patients who do not respond to conservative treatment, who have recurrent pressure sores and unstable scars, who lack material due to the extent of the sore or who lack adequate padding due to anatomical changes. ${ }^{1}$

The use of sophisticated neurosensory musculocutaneous flaps needs not only a very experienced plastic surgeon who is in continuous contact with a spinal cord injury unit, but also the help and assistance of an experienced nursing and rehabilitation team. Krupp and colleagues ${ }^{1}$ stated as early as 1983 the importance of adequate evaluations before performing such flaps.

\section{Results}

From 1981 to April 1989, 299 pressure sores in 206 patients were operated on by the second author. Ninety-one decubitus ulcers

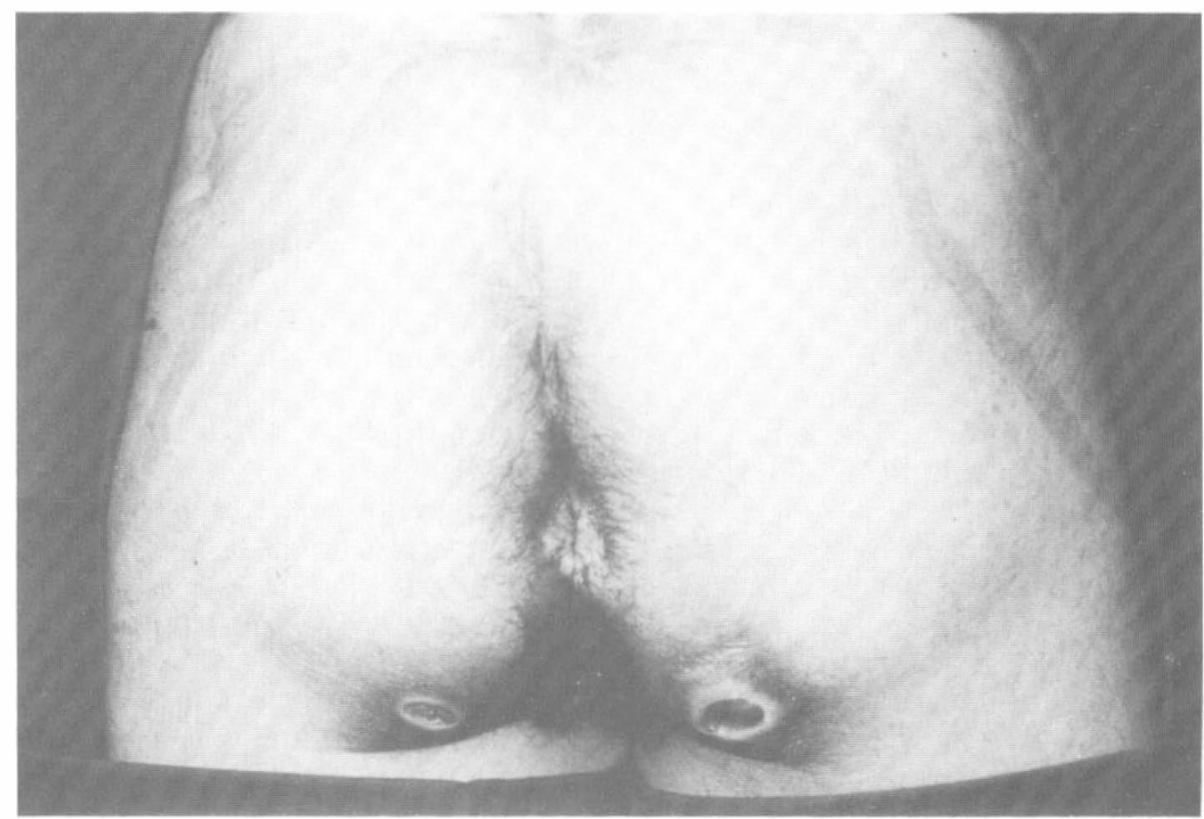

Figure 1 Typical recurrent ischial pressure sores in a meningomyelocele patient L3/4. The lines on the buttocks show the boundary between normalgesia and analgesia. 
were covered with muscle or musculocutaneous flaps. The TFL-flap was used 31 times. We review a consecutive series of 19 neurosensory TFL-flaps, including 6 flaps operated by the fourth author in 1980 . The age of the patients ranged from 13 to 49 years and about half of them were walking paraplegics who had had a meningomyelocele.

Nine flaps were primarily delayed due to problems such as obesity and skeletal deformity. On 14 occasions the TFL-flap was transposed laterally and 3 times medially. Twice an extended medial island flap with de-epithelialisation was used. Twelve pressure sores were primarily debrided and covered at a second operation by a neurosensory flap.

All living patients were reexamined personally by the plastic surgeons. In one patient with a recurrent ischial pressure sore, the lateral circumflex femoral artery could not be shown in the preoperative angiogram. Nevertheless a surgical exploration was done which unfortunately confirmed the missing main proximal vessel. Therefore this flap could not be raised and the pressure sore had to be covered with a local transposition flap.

\section{Early complications}

Wound dehiscence without necrosis was seen once. Three flaps showed a marginal necrosis of less than $1 \mathrm{~cm}$ in size. In 2 cases the flap tip was lost $(2-3 \mathrm{~cm})$, one of these cases having to be secondarily skin-grafted. Comparing the group of the 10 with delayed flaps with the 9 with non delayed flaps, it is obvious that marginal necroses can be better avoided if there is a delay. The patient who lost his flap-tip in the delay group had already shown an important reduction of blood supply during the delayed procedure.

There were 2 gluteal abscesses 4 and 6 months after the first operation in patients with multiple fistulas; healing was spontaneous, not being influenced by the delayed procedure.

In the 10 patients whose flaps were not delayed, 6 postoperative complications occurred and in the 9 patients with the delayed procedure only 3 complications were seen.

\section{Late follow up study}

One patient died 4 years postoperatively, with the flap healed completely and no signs of recurrence. The remaining 17 flaps could be seen and examined in March 1989. The average time since operation was 69 months, the maximum follow up time 110 months (Fig. 2).

Sensation. Nine flaps showed normal cutaneous sensation. Four flaps were hypoalgesic in the meningomyelocele patients, but this hypoalgesia was comparable to their former sensitivity in the lateral thigh. Three flaps showed patchy areas of analgesia; in one patient the flap was totally anaesthetic at the last $7 \mathrm{~cm}$. Ten flaps provided perception of the filling state of the rectum due to the vicinity of the end of the TFL-flap to the perineum and rectum. Two patients complained of marked hyperalgesia in or around the flap.

In 3 patients (in one patient even after 9 years) sensation within the flap was still localised to the lateral thigh. Younger patients learned to position their new sensory skin territories correctly in relation to the sitting area within some months but the others needed on average one to 2 years.

Nerve sprouting with extension of the sensitivity outside the TFL-flap could not be shown in any patient.

Recurrence. Two superficial and 2 deep recurrences on the transposed TFL-flap had to be noted, but 3 of these 4 recurrences were in the patient with the anaesthetic end of the flap and the other recurrence was in a patient with a hypalgesic TFL-flap.

Six new deep pressure ulcers in other sites than the covered ones occurred in the same follow up period in the pelvic region. Five of these new pressure sores occurred in the same patients who had already shown local recurrence. One patient with severe schizophrenia and catatony had one superficial and 2 deep local recurrences on his anaesthetic flap and additionally 2 new pressure sores in other sites than the pelvic region.

Reduced patient compliance is still a main risk factor for skin breakdown or recurrence. 


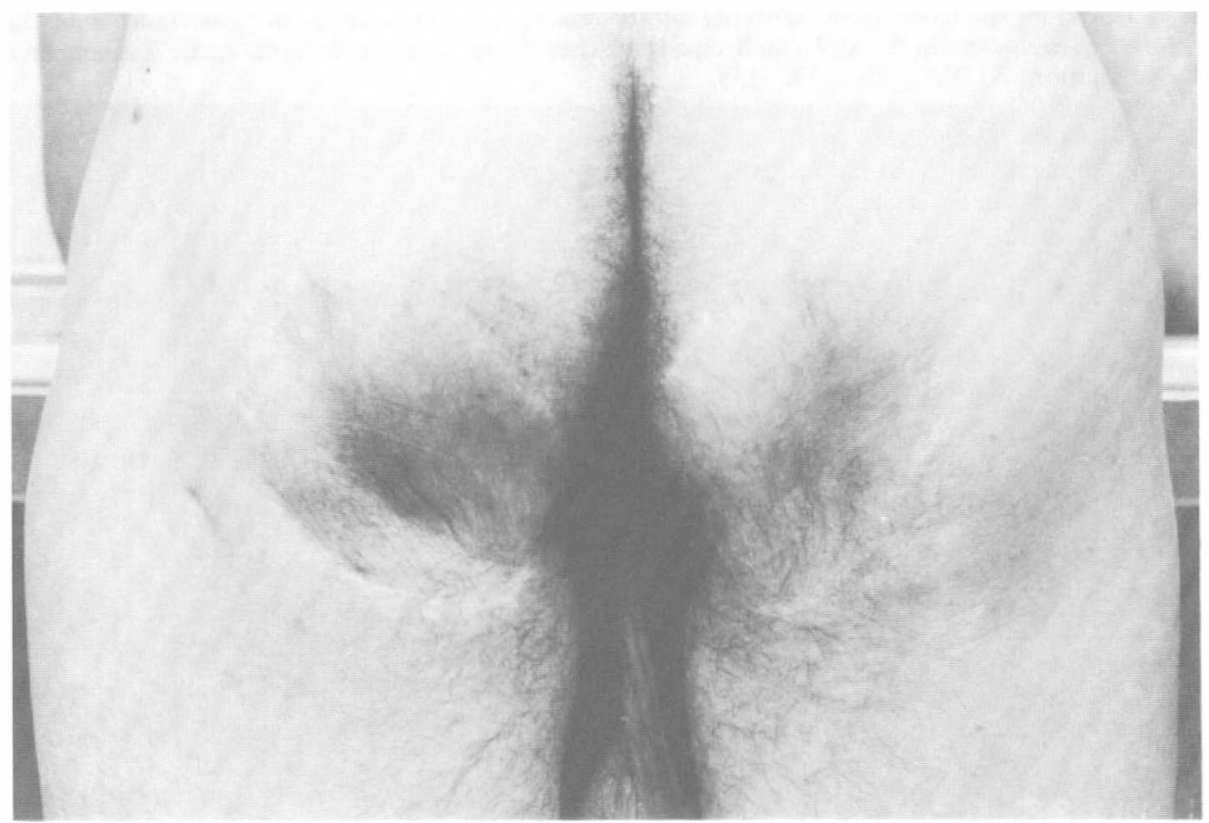

Figure 2 10-years-control of the first patient operated in 1980 and presented in Paraplegia 21: $123-4$, shows the medial island flaps without recurrence (proximally and distally de-epithelialised TFL-flaps).

\section{Discussion and conclusions}

The long interval between operation and follow up for all of the patients allows the following conclusions:

1 The extended tensor fasciae latae flap is technically quite demanding.

2 In some patients a two or three stage procedure cannot be avoided.

3 The donor site leaves an extensive scar, especially if it has to be covered with a skin graft.

4 Operation and time of hospitalisation is longer for long neurosensory TFL-flaps than for a local procedure.

5 Some patients complain about their new modified skin sensation, although this new sensation protects them against local recurrence.

Our long term follow up of neurosensory musculocutaneous tensor fasciae latae flaps proves clearly that:

1 No local recurrence on or around a neurosensory TFL-flap occurs in the long term evolution provided that the flap has complete cutaneous sensation.

2 An extended neurosensory TFL-flap can indicate the filling status of the rectum thus improving the quality of life considerably.

3 In most of the patients, sitting control and perception of the body scheme was also improved by the new sensation in the sitting area.

As a final conclusion we claim that if ever the neurological situation allows any neurosensory musculocutaneous flaps, such flaps should be used in paraplegic patients. Restoring protective sensation in decubitus predisposed regions prevents any local recurrence.

\section{References}

1 Krupp S, Kuhn W, Zaech GA (1983) The use of innervated flaps for the closure of ischial pressure sores. Paraplegia 21: 119-126. 
2 Kuhn W (1987) Möglichkeiten und Grenzen der Betreuung Querschnittgelähmter ausserhalb eines Zentrums der Schweiz. Schlussbericht 5. Alpenländisch-adriatisches Symposium für Internationale Zusammenarbeit in der Rehabilitation, AUVA, Wien: 130-133.

3 Longe RL (1986) Current concepts in clinical therapeutics: pressure sores. Clin Pharm 5: 669.

4 Luescher NJ (1989) Decubitalulcera der Beckenregion. Hans Huber, Bern.

5 Young JS, Burns PE, Bowen AM, McCutchen R (1982) Spinal Cord Injury Statistics. Good Samaritan Medical Center, Phoenix, Arizona.

6 Cochran JH, Edstrom LE, Dibbell DG (1981) Usefulness of the innervated tensor fascia lata flap in paraplegic patients. Ann Plast Surg 7: 286.

7 Coleman JJ III, Jurkiewicz MJ (1984) Methods of providing sensation to anesthetic areas. Ann Plast Surg 12: 177.

8 Daniel RK, Terzis JK, Cunningham DM (1967) Sensory skin flaps for coverage of pressure sores in paraplegic patients. Plast Reconstr Surg 58: 317.

9 Lister GD (1978) Use of an innervated skin graft to provide sensation to the reconstructed heel. Plast Reconstr Surg 62: 157.

10 Mackinnon S, Dellon AL, Patterson, GA, Gruss JS (1985) Medial antebrachial cutaneous-lateral femoral cutaneous neurotization to provide sensation to pressure-bearing areas in the paraplegic patient. Ann Plast Surg 6: 541 .

11 Mathes SJ, Buchanan RT (1979) Tensor fascia lata: neurosensory musculo-cutaneous free flap. Br J Plast Surg 32: 184.

12 Dibbell DG (1974) Use of a long island flap to bring sensation to the sacral area in young paraplegics. Plast Reconstr Surg 54: 220.

13 Nahai F (1980) The tensor fascia lata flap. Clin Plast Surg 7: 51.

14 Zufferey J, Doerfl J, Krupp S (1988) The anatomical basis for delaying the musculocutaneous tensor fascia lata flap with a distal extension. Eur J Plast Surg 11: 109.

15 Louie G, Mackinnon SE, Dellon AL, Patterson GA, Hunter DA (1987) Medial antebrachial cutaneouslateral femoral cutaneous neurotization in restoration of sensation to pressure-bearing areas in a paraplegic: a four-year follow-up. Ann Plast Surg 19: 572.

16 Krupp S, Milliet AC (1982) Why and when to delay TFL flaps. Chir Plastica 7: 23. 